\title{
Fast and Accurate Analysis of Complicated Metamaterial Structures Using a Low-Frequency Multilevel Fast Multipole Algorithm
}

\author{
Ö. Ergül ${ }^{*, \dagger} \quad$ L. Gürel ${ }^{*, \dagger}$
}

\begin{abstract}
We present efficient solutions of electromagnetics problems involving realistic metamaterial structures using a low-frequency multilevel fast multipole algorithm (LF-MLFMA). Ordinary implementations of MLFMA based on the diagonalization of the Green's function suffer from the low-frequency breakdown, and they become inefficient for the solution of metamaterial problems discretized with very small elements compared to the wavelength. We show that LF-MLFMA, which employs multipoles explicitly without diagonalization, significantly improves the solution of metamaterial problems in terms of both processing time and memory.
\end{abstract}

\section{INTRODUCTION}

Metamaterials are artificial structures that are constructed by periodically arranging unit cells, such as split-ring resonators (SRRs), as depicted in Figure 1. Due to their unusual but useful electromagnetic properties, metamaterials can be utilized in various applications [1], such as sub-wavelength focusing, cloaking, and designing improved antennas. Accurate simulations of metamaterials are essential in order to understand electromagnetic properties of those structures and to investigate novel designs before their actual realizations.

The multilevel fast multipole algorithm (MLFMA) is a powerful method, which enables accurate solutions of electromagnetics problems discretized with large numbers of unknowns [2]. Matrix-vector multiplications required by iterative solvers can be performed efficiently by MLFMA in $\mathcal{O}(N \log N)$ time using $\mathcal{O}(N \log N)$ memory, where $N$ is the number of unknowns. Three-dimensional realistic metamaterial structures involving hundreds of unit cells can be analyzed rigorously via MLFMA without any homogenization approximations [3]. Nevertheless, ordinary implementations of MLFMA based on the diagonalization of the Green's function are

\footnotetext{
*Department of Electrical and Electronics Engineering, Bilkent University, TR-06800, Bilkent, Ankara, Turkey.

${ }^{\dagger}$ Computational Electromagnetics Research Center (BiLCEM), Bilkent University, TR-06800, Bilkent, Ankara, Turkey.

e-mail: \{ergul,lgurel\}@ee.bilkent.edu.tr

tel.: +90 312 2905750, fax: +90 3122905755 .
}

not very suitable for the solution of metamaterial problems. Due to the low-frequency breakdown, clusters in the ordinary MLFMA cannot be very small compared to the wavelength [4]. On the other hand, metamaterials usually involve small details that must be discretized with small elements compared to the wavelength. Consequently, when the ordinary MLFMA is applied on metamaterial structures, the lowest-level clusters involve many discretization elements. This significantly increases the processing time and memory required for near-field interactions that must be calculated directly. Even the complexity of MLFMA can be more than $\mathcal{O}(N \log N)$ due to excessively large numbers of near-field interactions.

In this paper, we show that a low-frequency MLFMA (LF-MLFMA), which is based on using multipoles, provides more efficient solutions of metamaterial problems than the ordinary MLFMA. Without diagonalization, LF-MLFMA does not suffer from the low-frequency breakdown, and the number of levels can be chosen appropriately to increase the efficiency in terms of processing time and memory.

\section{MULTILEVEL FAST MULTIPOLE AL- GORITHM}

In MLFMA, interactions between discretization elements, i.e., basis and testing functions, are calculated in a group-by-group manner using the factorization of the homogeneous-space Green's function. A multilevel tree structure of $L$ levels is constructed by placing the object in a cubic box and recursively dividing the computational domain into subdomains (clusters). Using the tree structure, interactions between distant clusters are computed efficiently in three stages, namely, aggregation, translation, and disaggregation [2].

\subsection{Ordinary MLFMA}

In the ordinary form of MLFMA, radiated and incoming fields are represented by plane waves. For level $l=1,2, \ldots, L$, the number of samples (planewave directions) is $S_{l}=\left(T_{l}+1\right)^{2}$, where $T_{l}$ is 


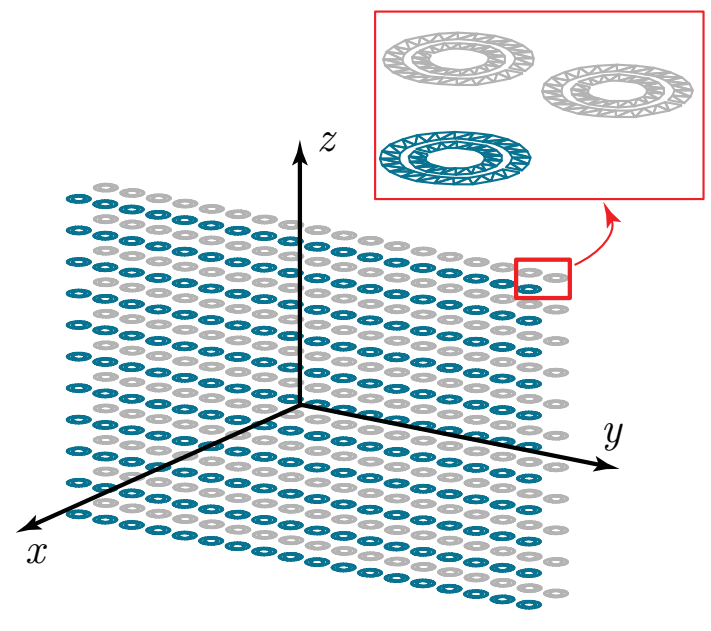

Figure 1: A 2-layer metamaterial wall involving $18 \times 11$ SRRs

the truncation number. Aggregations at the lowest level can be written as

$$
\left\{\boldsymbol{s}_{\theta}^{C}, \boldsymbol{s}_{\phi}^{C}\right\}=\sum_{n \in C} \boldsymbol{x}[n]\left\{\boldsymbol{s}_{\theta}^{n}, \boldsymbol{s}_{\phi}^{n}\right\}
$$

where $\boldsymbol{x}[n]$ represents coefficients provided by the iterative solver, $s_{\theta}^{C}$ and $s_{\phi}^{C}$ are arrays of $S_{1}$ elements containing the radiated field of a cluster $C$ at the lowest level, and $s_{\theta}^{n}$ and $s_{\phi}^{n}$ are arrays of $S_{1}$ elements containing the radiation pattern of the $n$th basis function inside $C$. For a cluster $C$ at level $l>1$,

$$
\begin{aligned}
& \left\{\boldsymbol{s}_{\theta}^{C}, \boldsymbol{s}_{\phi}^{C}\right\} \\
& \quad=\sum_{C^{\prime} \in C} \overline{\boldsymbol{\beta}}_{C^{\prime} \rightarrow C} \cdot \overline{\boldsymbol{\Gamma}}_{(l-1) \rightarrow l} \cdot\left\{\boldsymbol{s}_{\theta}^{C^{\prime}}, \boldsymbol{s}_{\phi}^{C^{\prime}}\right\},
\end{aligned}
$$

where $\overline{\boldsymbol{\beta}}_{C^{\prime} \rightarrow C}$ is a $S_{l} \times S_{l}$ diagonal matrix containing exponential (plane-wave-to-plane-wave) shift functions between cluster centers and $\overline{\boldsymbol{\Gamma}}_{(l-1) \rightarrow l}$ is a $S_{l} \times S_{l-1}$ sparse interpolation matrix to increase the sampling rate from level $(l-1)$ to $l$.

In the translation stage, radiated fields of clusters are translated into incoming fields for other clusters. For a cluster $C$ at level $l$,

$$
\left\{\boldsymbol{g}_{\theta}^{C}, \boldsymbol{g}_{\phi}^{C}\right\}=\sum_{C^{\prime} \in \mathcal{F}\{C\}} \overline{\boldsymbol{\alpha}}_{C^{\prime} \rightarrow C} \cdot\left\{\boldsymbol{s}_{\theta}^{C^{\prime}}, \boldsymbol{s}_{\phi}^{C^{\prime}}\right\},
$$

where $\boldsymbol{g}_{\theta}^{C}$ and $\boldsymbol{g}_{\phi}^{C}$ are arrays of $S_{l}$ elements containing the incoming field to the center of $C, \mathcal{F}\{C\}$ represents clusters that are far from $C$, and $\overline{\boldsymbol{\alpha}}_{C^{\prime} \rightarrow C}$ is a $S_{l} \times S_{l}$ diagonal translation matrix.

In the disaggregation stage, total incoming fields at cluster centers are calculated from the top of the tree structure to the lowest level. For a cluster $C$ at level $(l-1)$, the total incoming field can be written as

$$
\begin{aligned}
& \left\{\boldsymbol{g}_{\theta}^{C+}, \boldsymbol{g}_{\phi}^{C+}\right\}=\left\{\boldsymbol{g}_{\theta}^{C}, \boldsymbol{g}_{\phi}^{C}\right\} \\
& \quad+\overline{\boldsymbol{\Delta}}_{l \rightarrow(l-1)} \cdot \overline{\boldsymbol{\beta}}_{\mathcal{P}\{C\} \rightarrow C} \cdot\left\{\boldsymbol{g}_{\theta}^{\mathcal{P}\{C\}}, \boldsymbol{g}_{\phi}^{\mathcal{P}\{C\}}\right\}
\end{aligned}
$$

where $\mathcal{P}\{C\}$ represents the parent cluster and $\overline{\boldsymbol{\Delta}}_{l \rightarrow(l-1)}$ is a $S_{l-1} \times S_{l}$ sparse anterpolation (transpose interpolation) matrix to decrease the sampling rate from level $l$ to $(l-1)$. Finally, at the lowest level, incoming fields are received by testing functions as

$$
\sum_{n \in \mathcal{F}\{m\}} \overline{\boldsymbol{Z}}[m, n] \boldsymbol{x}[n] \propto \boldsymbol{f}_{\theta}^{m} \cdot \boldsymbol{g}_{\theta}^{C+}+\boldsymbol{f}_{\phi}^{m} \cdot \boldsymbol{g}_{\phi}^{C+},
$$

where $\boldsymbol{f}_{\theta}^{m}$ and $\boldsymbol{f}_{\phi}^{m}$ are arrays of $S_{1}$ elements containing the receiving pattern of the $m$ th testing function inside $C$.

In MLFMA, the sampling rate depends on the cluster size as measured by the wavelength $(\lambda)$. For level $l=1,2, \ldots, L$, the number of samples can be approximated as $S_{l}=4^{(l-1)} S_{1}$, where $S_{1}=\mathcal{O}(1)$. Considering the number of clusters $N_{l} \approx 4^{(1-l)} N_{1}$, where $N_{1}=\mathcal{O}(N)$, the computational cost of the ordinary MLFMA is $\mathcal{O}(N)$ per level. For ordinary structures discretized with $\lambda / 10$ triangles, $L=\mathcal{O}(\log N)$, and there are $\mathcal{O}(N)$ near-field interactions that must be calculated directly. Hence, for those problems, the overall complexity of the ordinary MLFMA is $\mathcal{O}(N \log N)$. In the case of metamaterials or similar structures, however, the efficiency of the ordinary MLFMA may deteriorate significantly.

\subsection{Low-Frequency MLFMA}

In LF-MLFMA, radiated and incoming fields are represented explicitly by multipoles. For level $l=1,2, \ldots, L$, the number of multipoles is $M_{l}=$ $\left(T_{l}+1\right)^{2}$, where $T_{l}$ is the truncation number. Aggregations at the lowest level can be written as

$$
\left\{\boldsymbol{s}_{\theta}^{C}, \boldsymbol{s}_{\phi}^{C}, \boldsymbol{s}_{r}^{C}, \boldsymbol{s}_{s}^{C}\right\}=\sum_{n \in C} \boldsymbol{x}[n]\left\{\boldsymbol{s}_{\theta}^{n}, \boldsymbol{s}_{\phi}^{n}, \boldsymbol{s}_{r}^{n}, \boldsymbol{s}_{s}^{n}\right\} .
$$

As opposed to the diagonal form in (1), the radial components of the vector-potential part, as well as the scalar-potential part are required in (6). For a cluster $C$ at level $l>1$,

$$
\begin{aligned}
& \left\{\boldsymbol{s}_{\theta}^{C}, \boldsymbol{s}_{\phi}^{C}, \boldsymbol{s}_{r}^{C}, \boldsymbol{s}_{s}^{C}\right\} \\
& \quad=\sum_{C^{\prime} \in C} \overline{\boldsymbol{\beta}}_{C^{\prime} \rightarrow C} \cdot\left\{\boldsymbol{s}_{\theta}^{C^{\prime}}, \boldsymbol{s}_{\phi}^{C^{\prime}}, \boldsymbol{s}_{r}^{C^{\prime}}, \boldsymbol{s}_{s}^{C^{\prime}}\right\}
\end{aligned}
$$

where $\overline{\boldsymbol{\beta}}_{C^{\prime} \rightarrow C}$ is a $M_{l} \times M_{l-1}$ dense matrix containing multipole-to-multipole shift functions. Without diagonalization, translations also involve dense 
matrix-vector multiplications. For a cluster $C$ at level $l$,

$$
\begin{aligned}
& \left\{\boldsymbol{g}_{\theta}^{C}, \boldsymbol{g}_{\phi}^{C}, \boldsymbol{g}_{r}^{C}, \boldsymbol{g}_{s}^{C}\right\} \\
& \quad=\sum_{C^{\prime} \in \mathcal{F}\{C\}} \overline{\boldsymbol{\alpha}}_{C^{\prime} \rightarrow C} \cdot\left\{\boldsymbol{s}_{\theta}^{C^{\prime}}, \boldsymbol{s}_{\phi}^{C^{\prime}}, \boldsymbol{s}_{r}^{C^{\prime}}, \boldsymbol{s}_{s}^{C^{\prime}}\right\},
\end{aligned}
$$

where $\overline{\boldsymbol{\alpha}}_{C^{\prime} \rightarrow C}$ is a $M_{l} \times M_{l}$ dense matrix containing multipole-to-multipole translation functions. Then, the total incoming field for a cluster $C$ at level $(l-1)$ can be calculated as

$$
\begin{aligned}
\left\{\boldsymbol{g}_{\theta}^{C+}\right. & \left., \boldsymbol{g}_{\phi}^{C+}, \boldsymbol{g}_{r}^{C+}, \boldsymbol{g}_{s}^{C+}\right\} \\
& =\left\{\boldsymbol{g}_{\theta}^{C}, \boldsymbol{g}_{\phi}^{C}, \boldsymbol{g}_{r}^{C}, \boldsymbol{g}_{s}^{C}\right\}+\overline{\boldsymbol{\beta}}_{\mathcal{P}\{C\} \rightarrow C} \\
& \cdot\left\{\boldsymbol{g}_{\theta}^{\mathcal{P}\{C\}+}, \boldsymbol{g}_{\phi}^{\mathcal{P}\{C\}+}, \boldsymbol{g}_{r}^{\mathcal{P}\{C\}+}, \boldsymbol{g}_{s}^{\mathcal{P}\{C\}+\}}\right\}
\end{aligned}
$$

Finally, at the lowest level, incoming fields are received by testing functions as

$$
\begin{aligned}
\sum_{n \in \mathcal{F}\{m\}} \overline{\boldsymbol{Z}}[m, n] \boldsymbol{x}[n] & \propto \boldsymbol{f}_{\theta}^{m} \cdot \boldsymbol{g}_{\theta}^{C+}+\boldsymbol{f}_{\phi}^{m} \cdot \boldsymbol{g}_{\phi}^{C+} \\
& +\boldsymbol{f}_{r}^{m} \cdot \boldsymbol{g}_{r}^{C+}+\boldsymbol{f}_{s}^{m} \cdot \boldsymbol{g}_{s}^{C+}
\end{aligned}
$$

for $m \in C$.

Using LF-MLFMA, the cluster size is not restricted, and we are able to recursively divide the object into sub-clusters, which can be much smaller than the wavelength. This way, the number of nearfield interactions is always bounded with $\mathcal{O}(N)$ complexity. In addition, for metamaterial structures with dimensions of several wavelengths, the number of multipoles required for far-field interactions is almost constant. Then, the computational cost of LF-MLFMA is dominated by computations at the lowest level with $\mathcal{O}(N)$ complexity. We emphasize that LF-MLFMA may not be appropriate for large-scale problems since the multipole representation becomes inefficient for large clusters. For those problems, we employ a broadband implementation of MLFMA [5], where the ordinary MLFMA and LF-MLFMA are used at higher and lower levels, respectively, of the same tree structure.

\section{RESULTS}

As an example, we consider the solution of scattering problems involving a metamaterial structure depicted in Figure 1. A 2-layer metamaterial wall is constructed by periodically arranging $2 \times 18 \times 11$ SRRs. A single SRR has dimensions in the order of microns and resonates at about $100 \mathrm{GHz}$ when embedded into a homogeneous host medium with a relative permittivity of 4.8 [3]. The incident field is generated by a Hertzian dipole located at $x=1.2 \mathrm{~mm}$. For numerical solutions, surfaces

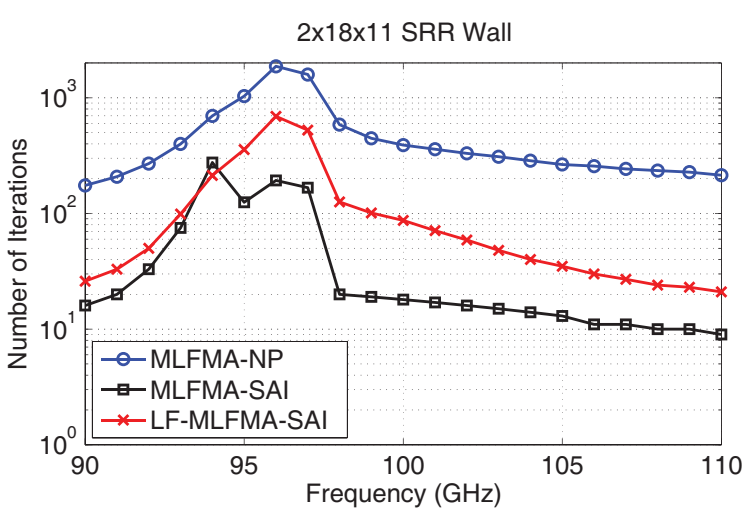

(a)

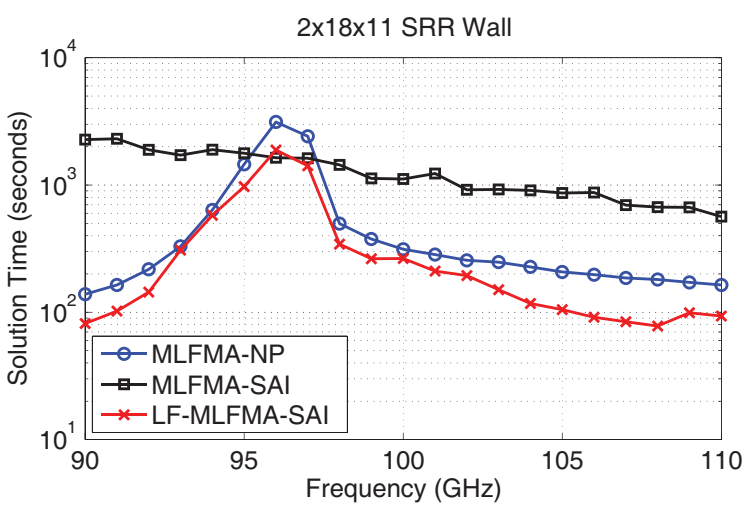

(b)

Figure 2: Solutions of scattering problems involving a 2-layer SRR wall depicted in Figure 1. (a) Number of GMRES iterations $\left(10^{-3}\right.$ residual error $)$ and (b) solution time are plotted with respect to frequency.

are discretized with $\lambda / 100$ triangles, where $\lambda$ is the wavelength in the host medium at $100 \mathrm{GHz}$. Such a dense discretization is required for accurate modelling of SRRs that involve small details with respect to the wavelength. Problems are formulated with the electric-field integral equation discretized with the Rao-Wilton-Glisson functions, and matrix equations involving 32,472 unknowns are solved iteratively by the generalized-minimal residual (GMRES) algorithm without restart. Matrix-vector multiplications are performed by the ordinary MLFMA and LF-MLFMA with two digits of accuracy. Iterative solutions are also accelerated by the sparse-approximate-inverse (SAI) preconditioner constructed from the near-field interactions without filtering.

Figures 2 and 3 present the number of iterations for $10^{-3}$ residual error, solution time, total time, and memory required for solutions with respect to frequency from $90 \mathrm{GHz}$ to $110 \mathrm{GHz}$. The 


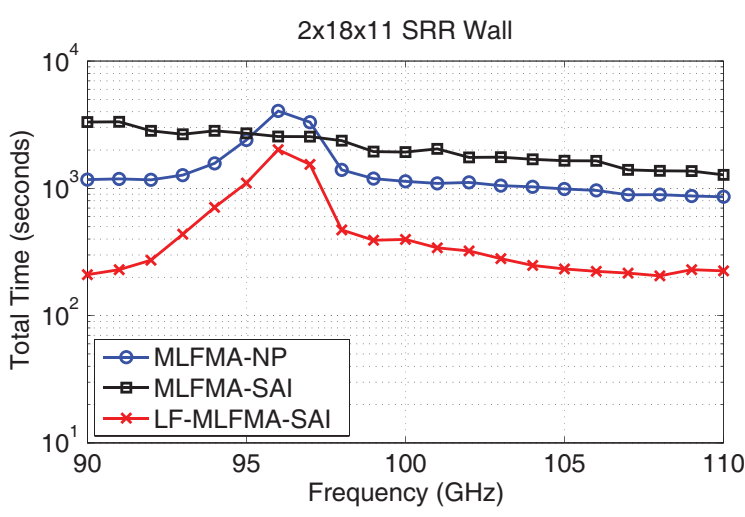

(a)

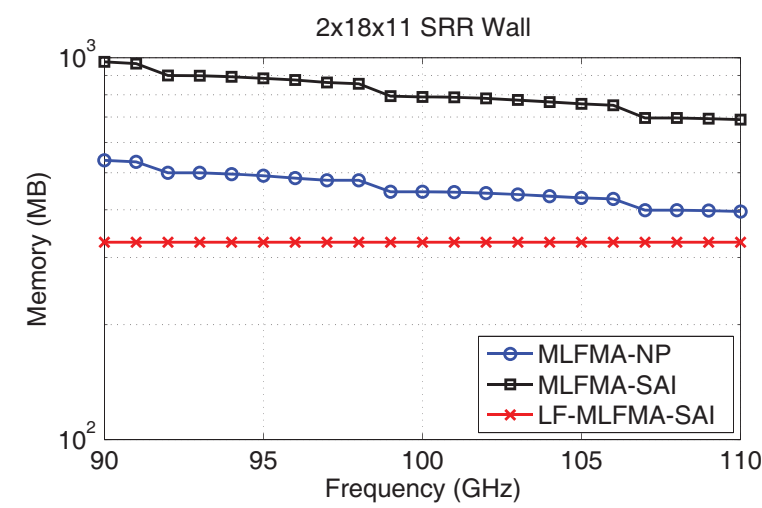

(b)

Figure 3: Solutions of scattering problems involving a 2-layer SRR wall depicted in Figure 1. (a) Total time including setup and solution times and (b) memory required for solutions are plotted with respect to frequency.

number of iterations peaks at $95 \mathrm{GHz}$ due to a numerical resonance. Considering only the ordinary MLFMA, Figure 2(a) shows that the SAI preconditioner reduces the number of iterations significantly compared to the no-preconditioner (NP) case. On the other hand, as depicted in Figure 2(b), the solution time is not reduced due to the additional factorization cost of the preconditioner. In other words, reducing the number of iterations does not necessarily accelerate solutions via the ordinary MLFMA. We also observe in Figure 2(a) that using LF-MLFMA, instead of the ordinary MLFMA, increases the number of iterations since the number of near-field interactions used to construct the preconditioner is smaller in LF-MLFMA, compared to the ordinary MLFMA. On the other hand, Figure 2(b) shows that, except for $96 \mathrm{GHz}$, the most efficient solutions are provided by LF-MLFMA accelerated via SAI. Superior performance of LF-MLFMA becomes more apparent in terms of the total time depicted in Figure 3(a), which includes the setup time dominated by the near-field interactions in addition to the solution time. Finally, Figure 3(b) shows that, in addition to faster solutions, LF-MLFMA requires less memory than the ordinary MLFMA.

\section{CONCLUSION}

Metamaterial structures involving small details with respect to the wavelength can be analyzed more efficiently via LF-MLFMA using multipoles instead of the ordinary MLFMA using plane waves. We show that accelerated iterative convergence provided by robust preconditioning techniques may not be sufficient to reduce the processing time without overcoming the major bottleneck, i.e., lowfrequency breakdown of MLFMA.

\section{Acknowledgments}

This work was supported by the Turkish Academy of Sciences in the framework of the Young Scientist Award Program (LG/TUBA-GEBIP/20021-12), by the Scientific and Technical Research Council of Turkey (TUBITAK) under Research Grants 105E172 and 107E136, and by contracts from ASELSAN and SSM.

\section{References}

[1] N. Engheta and R. W. Ziolkowski, "A positive future for double-negative metamaterials," IEEE Trans. Microw. Theory Tech., vol. 53, no. 4, pp. 1535-1556, Apr. 2005.

[2] J. Song, C.-C. Lu, and W. C. Chew, "Multilevel fast multipole algorithm for electromagnetic scattering by large complex objects," IEEE Trans. Antennas Propag., vol. 45, no. 10, pp. 1488-1493, Oct. 1997.

[3] L. Gürel, Ö. Ergül, and A. Ünal, "Accurate analysis of metamaterials involving finite arrays of split-ring resonators and thin wires," in Proc. Progress in Electromagnetics Research Symp. (PIERS), 2007, pp. 470-473.

[4] J.-S. Zhao and W. C. Chew, "Three dimensional multilevel fast multipole algorithm from static to electrodynamic," Microw. Opt. Technol. Lett., vol. 26, no. 1, pp. 43-48, Jul. 2000.

[5] L. J. Jiang and W. C. Chew, "A mixed-form fast multipole algorithm," IEEE Trans. Antennas Propag., vol. 53, no. 12, pp. 4145-4156, Dec. 2005. 